\title{
Prescription des antibiotiques en odontologie et stomatologie Recommandations
}

\author{
AGENCE FRANÇAISE DE SÉCURITÉ SANITAIRE \\ DES PRODUITS DE SANTÉ (AFSSAPS)
}

\section{GROUPE DE TRAVAIL}

Dr VEYSSIER Pierre, Président, Infectiologue, membre du GTA, Compiègne

Dr NOUYRIGAT Emmanuel, Responsable et Chargé de Projet, Afssaps

Dr BEN SLAMA Lotfi, Stomatologue, Paris

Dr BENSAHEL Jean-Jacques, Chirurgien-Dentiste, Nice

Pr BERTRAND Jacques-Charles, Stomatologue,

Chirurgien Maxillo-Facial, Paris

Pr BOY-LEFEVRE Marie-Laure, Chirurgien-Dentiste,

Charenton-Le-Pont

Dr DERSOT Jean-Marc, Parodontologue, Paris

\section{GROUPE DE LECTURE}

Dr ARRETO Charles-Daniel, Chirurgien-Dentiste, Paris

Dr BARSOTTI Odile, Chirurgien-Dentiste, Lyon

Dr BOUCHARD Philippe, Chirurgien-Dentiste, Paris

Pr BOUVET Anne, Microbiologiste, Paris

Dr BRU Jean-Pierre, Infectiologue, Annecy

Pr CHIDIAC Christian, Infectiologue, Lyon

Pr DE MELLO Gilbert, Chirurgien-Dentiste, Rennes

Pr DEVEAUX Etienne, Chirurgien-Dentiste, Lille

Pr DURAN Damien, Chirurgien-Dentiste, Toulouse

Dr ETIENNE Daniel, Parodontologue, Paris

Pr FEKI Ahmed, Chirurgien-Dentiste, Strasbourg

Dr FOURNIER Sandra, Infectiologue, Paris

Pr GARRE Michel, Infectiologue, Brest

Dr GIOVANNOLI Jean-Louis, Chirurgien-Dentiste, Paris

Dr IMBERT Simone, Stomatologue, Paris

Dr JOACHIM Frédéric, Chirurgien-Dentiste, Lille

Dr JORRY Jean-François, Parodontologue, Tonnerre

Dr LAIK Louis, Chirurgien-Dentiste, Pegomas

\section{COMITE DE VALIDATION}

Pr BOUVENOT Gilles, Président,Thérapeutique, Marseille

Pr BERGMANN Jean-François, Vice-Président, Thérapeutique, Paris

Pr CAULIN Charles, Président de la Commission d'AMM, Paris

Pr DUPUIS Bernard, Président de la Commission

de Transparence, Lille

Dr RICHÉ Christian, Président de la Commission de

Pharmacovigilance, Brest

Pr AUBIER Michel, Pneumologue, Clichy

Pr BANNWARTH Bernard, Pharmacologue, Rhumatologue,

Bordeaux

Dr CAMELLI Bruno, Généraliste, Paris

Pr FUNCK-BRENTANO Christian, Pharmacologue Clinicien,

Cardiologue, Paris
Pr DUBREUIL Luc, Microbiologiste, Lille

Dr LESCLOUS Philippe, Chirurgien-Dentiste, Ivry-sur-Seine

Dr RAGOT Jean-Pierre, Stomatologue, Chirurgien maxillo-facial,

Paris

Dr SENNEVILLE Eric, Infectiologue, Tourcoing

Pr SIXOU Michel, Chirurgien-Dentiste, Toulouse

Dr DENIS Catherine, Afssaps

Dr DUMARCET Nathalie, Afssaps

Dr KONOPKA Claude-Annick, Afssaps

Dr PELLANNE Isabelle, Afssaps

Dr LEMAITRE Philippe, Parodontologue, Nantes

Pr LEPORT Catherine, Infectiologue, Paris

Dr LEROY Olivier, Infectiologue, Tourcoing

Dr MAES Jean-Michel, Stomatologue, Chirurgien maxillo-facial, Lille

Pr MONDIE Jean-Michel, Stomatologue, Chirurgien

maxillo-facial, Clermont-Ferrand

Dr MORA Francis, Parodontologue, Bordeaux

Dr MOUTON Christian, Chirurgien-Dentiste, Lembras

Dr OTTAVI Philippe, Stomatologue, Le Mans

Dr PEAN Yves, Biologiste, Paris

Pr POTEL Gilles, Thérapeutique, Nantes

Dr ROCHE Yvon, Chirurgien-Dentiste, Paris

Dr SAMAKH Paul, Parodontologue, Paris

Pr SAMSON Jacky, Stomatologue, Chirurgien maxillo-facial,

Genève

Dr SIXOU Jean-Louis, Chirurgien-Dentiste, Rennes

Pr SOUSSY Claude-James, Microbiologiste, Créteil

Pr LE JEUNNE Claire, Thérapeutique, Paris

Pr PETIT Michel, Psychiatre, Sotteville-lès-Rouen

Dr REVEILLAUD Olivier, Généraliste, Paris

Pr THERY Claude, Cardiologue, Lille

Dr TREMOLIERES François, Infectiologue, Interniste,

Mantes-la-Jolie

Dr WONG Olivier, Généraliste, Paris

Dr DENIS Catherine, Afssaps

Dr DUMARCET Nathalie, Afssaps

Dr NOUYRIGAT Emmanuel, Afssaps

Dr PELLANNE Isabelle, Afssaps

Dr ROSTOCKER Guy, Afssaps médecine

buccale

chirurgie

buccale

VOL. $9, \mathrm{~N}^{\circ} 1$

2003

page 49

Nous remercions l'Agence Française de Sécurité Sanitaire des Produits de la Santé de nous avoir autorisés à reproduire ce texte. L'argumentaire et les références bibliographiques de ces recommandations sont disponibles sur le site internet de l'Afssaps: www.afssaps.sante.fr, dans la rubrique « Documentation et publications ". 
médecine

buccale chirurgie buccale

VOL. $9, \mathrm{~N}^{\circ} 1$ 2003

page 50

\section{METHODE GENERALE}

L'ordonnance n 96-345 du 24 avril 1996 relative à la maîtrise médicalisée des dépenses de soins a confié l'Agence Française de Sécurité Sanitaire des Produits de Santé (Afssaps) la mission d'établir les recommandations de bonne pratique et les références médicales, concernant le médicament. Elle stipule d'autre part que les recommandations de bonne pratique et références existantes doivent être régulièrement actualisées, en fonction des données nouvelles de la science.

C'est dans ce contexte que l'Afssaps propose une actualisation des recommandations de bonne pratique et références : «Prescription des antibiotiques en odontologie et stomatologie », établies par l'ANDEM en 1996.

Ces recommandations définissent une stratégie médicale optimale en fonction de l'état actuel des connaissances et précisent ce qui est utile ou inutile, voire dangereux, de faire dans une situation clinique donnée.

Ces recommandations résultent de l'analyse des données actuelles de la science issues de la littérature, et prend en compte les évaluations réalisées pour délivrer l'autorisation de mise sur le marché (AMM) des médicaments concernés, apprécier le service médical rendu (SMR) et élaborer les fiches de transparence. Les sociétés savantes ont été consultées (Conseil National de l'Ordre des Chirurgiens-Dentistes, Association de Parodontologie Clinique et d'Implantologie, Collège Français d'Orthopédie MandibuloFaciale, Société Française de Stomatologie et de Chirurgie Maxillo-Faciale, Société Française de Parodontologie, Société Française d'Orthopédie Dento-Faciale, Société Odontologique de Paris, Académie Nationale de Chirurgie Dentaire, Association Universitaire de Parodontologie, Collège Français de Biomatériaux Dentaires, Société Française d'Endodontie, Société Française de Gérodontologie, Société Française de Pédodontie, Association Dentaire Française) pour proposer des représentants susceptibles de participer aux groupes.

Le groupe de travail constitué par l'Afssaps a regroupé des experts de compétence (chirurgie dentaire, stomatologie, parodontologie, chirurgie maxillo-faciale, infectiologie, microbiologie), de mode d'exercice (hospitalo-universitaires, hospitaliers ou libéraux) et d'origine géographique divers. Le groupe de travail comprenait un président qui a dirigé le groupe et collecté les avis de l'ensemble des membres, un chargé de projet qui, en collaboration directe avec le président, a analysé la littérature et rédigé le document, et des représentants de l'Afssaps.

La recherche bibliographique a été réalisée par interrogation systématique des banques de données Medline, Pascal, HealthStar, CabHealth et Bibliodent. Elle a identifié préférentiellement les recommandations thérapeutiques, les conférences de consensus, les essais cliniques, les méta-analyses, les analyses de décisions et les revues de synthèse, publiés en langue française ou anglaise après 1996.

La bibliographie obtenue par voie automatisée a été complétée par une recherche manuelle. Celle-ci a consisté en une analyse des sommaires de revues générales (British Medical Journal, JAMA, Lancet, New England Journal of Medicine) de début juin 2000 à fin février 2001. De plus, les listes de références citées dans les articles déjà identifiés ont été consultées. Enfin, les membres du groupe de travail et du groupe de lecture ont pu transmettre d'autres articles.

La recherche bibliographique automatisée était basée sur les mots clés suivants :

dentistry / mouth diseases / tooth diseases / jaw diseases / antibiotics / antibiotic prophylaxis / drug resistance, microbial.

Au total, 331 références ont été sélectionnées et analysées. Parmi elles, 259 références ont été utilisées pour l'élaboration du texte, dont 58 appelées dans le texte et 201 indexées en bibliographie complémentaire.

L'argumentaire et les recommandations de ce travail ont été établis par le groupe selon la méthodologie proposée par I'ANAES (ANAES : Les recommandations pour la pratique clinique - Base méthodologique pour leur réalisation en France- 1999 ; Guide d'analyse de la littérature et gradations des recommandations - 2000). Chaque article a été analysé en appréciant la 
qualité méthodologique des études, afin d'affecter à chacun un niveau de preuve scientifique. Pour ce faire des grilles de lecture destinées à apprécier la qualité méthodologique et le niveau de preuve scientifique des documents ont été utilisées.

Les grades $A, B$, et $C$ sont attribués aux recommandations selon le niveau de preuve scientifique attribué aux études sur lesquelles elles reposent (cf Tableau infra). Lorsque les données de la littérature sont insuffisantes ou incomplètes, les recommandations sont basées sur un accord professionnel pour prendre en compte l'état des pratiques et les opinions d'experts.

Le texte a été soumis à un groupe de lecture avant d'être finalisé. Le groupe de lecture était composé d'experts de compétence, de mode d'exercice et d'origine géographique divers. Les experts de ce groupe de lecture, consultés par courrier, ont apprécié la qualité méthodologique et la validité scientifique du contenu, ainsi que la lisibilité, la faisabilité et l'applicabilité du texte. Leurs remarques ont été transmises à l'ensemble du groupe de travail qui a pu modifier son texte et a validé le document final.

Le texte a ensuite été soumis à l'avis du Comité de Validation des Recommandations et Références Médicales de l'Afssaps.

\section{RECOMMANDATIONS}

\section{Introduction}

Afin de limiter la survenue d'effets indésirables et l'émergence de plus en plus fréquente de résistances bactériennes, la prescription des antibiotiques doit être réservée aux seules situations cliniques où leur efficacité a été démontrée.

Pour l'élaboration de ces recommandations en matière d'antibiothérapie, sont pris en compte les données d'épidémiologie microbienne et le spectre des antibiotiques. Ainsi, il est possible que certains antibiotiques ayant I'AMM ne soient pas recommandés dans ce texte.

Le choix des antibiotiques pour le traitement des infections bucco-dentaires doit être fait en fonction des bactéries pathogènes supposées présentes au cours d'une pathologie donnée, et du spectre d'activité antibactérienne et de la pharmacocinétique des antibiotiques. II doit aussi tenir compte du critère de gravité de la pathologie et des antécédents du patient. Les prélèvements microbiologiques ne sont pas justifiés en pratique courante dans la majorité des cas.

La stratégie générale de prescription des antibiotiques proposée dans cette recommandation repose sur un accord professionnel.
Niveau de preuve scientifique des études

Niveau 1 :

- Essais comparatifs randomisés de forte puissance

- Méta-analyse d'essais comparatifs randomisés

- Analyse de décision basée sur des études bien menées

Niveau 2 :

- Essais comparatifs randomisés de faible puissance

Etudes comparatives non randomisées bien menées

Etudes de cohorte

Niveau 3 :

- Etudes cas-témoin

Niveau 4 :

- Etudes comparatives comportant des biais importants

- Etudes rétrospectives

Séries de cas

Etudes épidémiologiques descriptives (transversale, longitudinale)

\section{Force des recommandations (grade)}

A

Preuve scientifique établie

B

Présomption scientifique

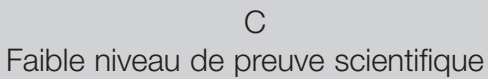

médecine

buccale

chirurgie

buccale

VOL. $9, \mathrm{~N}^{\circ} 1$ 2003

page 51 
médecine

buccale

chirurgie

buccale

VOL. $9, \mathrm{~N}^{\circ} 1$ 2003

page 52

\section{Notion de sujet à risque d'infection}

Les sujets sans facteur de risque ni terrain particulier sont des sujets considérés sains, sans risque infectieux reconnu ; certaines cardiopathies sont définies «sans risque d'endocardite infectieuse».

Deux types de sujets à risque infectieux sont définis :

- risque $A$ : risque d'infection identifiée localement et/ou de surinfection générale (septicémie). Ce risque concerne les sujets transplantés ou greffés (excepté les patients sous ciclosporine seule), les sujets immunodéprimés, les sujets atteints d'une pathologie chronique non contrôlée et les sujets dénutris.

- risque $B$ : risque d'infection liée à une localisation secondaire de la bactérie, c'est-à-dire à un nouveau foyer infectieux situé à distance du foyer primaire (endocardite infectieuse, infection sur prothèse articulaire). Ce risque concerne les sujets présentant une cardiopathie définie «à risque d'endocardite infectieuse » et certains sujets porteurs de prothèse.

\section{Antibiothérapie curative systémique}

\subsection{Indications de l'antibiothérapie}

\subsubsection{Sujets considérés sains}

Une antibiothérapie est recommandée chez les sujets considérés sains dans les pathologies suivantes : l'abcès périapical, la gingivite ulcéro-nécrotique, les parodontites agressives, la parodontite réfractaire, la péricoronarite, les cellulites (excepté la forme chronique), les ostéites infectieuses (excepté l'alvéolite sèche), les stomatites bactériennes et les infections bactériennes des glandes salivaires (Accord professionnel).

En ce qui concerne la parodontite de l'adulte, un traitement antibiotique, de préférence en monothérapie, pourra être prescrit en deuxième intention, en traitement adjuvant au traitement mécanique (Accord professionnel).

L'intérêt d'une antibiothérapie n'est pas reconnu chez les sujets considérés sains dans les patho- logies suivantes : la desmodontite apicale, les traumatismes alvéolo-dentaires, la parododontite chronique et au cours de la régénération tissulaire parodontale (Accord professionnel).

Une antibiothérapie n'est pas justifiée chez les sujets considérés sains dans les pathologies suivantes : les caries, les pulpopathies, leurs complications chroniques et la nécrose pulpaire, les gingivites chroniques, l'abcès parodontal, les accidents d'éruption des dents temporaires, les cellulites chroniques, les alvéolites sèches et lors des péri-implantites (Accord professionnel).

\subsubsection{Sujets à risque d'infection}

Une antibiothérapie est recommandée chez les sujets à risque $\mathrm{A}$ ou $\mathrm{B}$ dans les pathologies suivantes : l'abcès périapical, la nécrose pulpaire, les traumatismes alvéolo-dentaires compliqués, la gingivite ulcéro-nécrotique, les parodontites agressives, la parodontite chronique, la parodontite réfractaire, la péricoronarite, l'abcès parodontal, les cellulites, les ostéites (non justifié pour l'alvéolite sèche chez le sujet à risque A), les stomatites bactériennes et les infections bactériennes des glandes salivaires.

Elle est également recommandée chez le sujet à risque A lors des péri-implantites et au cours de la régénération tissulaire parodontale; ces actes sont contre-indiqués chez les sujets à risque $\mathrm{B}$. Elle est également recommandée chez le sujet à risque $\mathrm{B}$ pour la desmodontite apicale, la gingivite chronique, les gingivites associées à des maladies systémiques ou à la prise de médicaments, les accidents d'éruption des dents temporaires et l'alvéolite sèche (Accord professionnel).

L'intérêt d'une antibiothérapie n'est pas reconnu chez les sujets à risque $A$ ou $B$ dans les pathologies suivantes: les pulpites irréversibles, les complications périradiculaires chroniques, et les traumatismes alvéolo-dentaires simples.

Elle n'est également pas validée chez le sujet à risque $\mathrm{A}$ pour la gingivite chronique, les gingivites associées à des maladies systémiques ou à la prise de médicaments, et les accidents d'éruption des dents temporaires (Accord professionnel). 
Une antibiothérapie n'est pas justifiée chez le sujet à risque $A$ ou $B$ dans les caries et les pulpites transitoires réversibles.

Elle n'est également pas justifiée chez le sujet à risque $A$ dans les alvéolites sèches (Accord professionnel).

\subsection{Choix des antibiotiques}

\subsubsection{Infections de sévérité moyenne}

Dans les infections de sévérité moyenne, les antibiotiques recommandés en première intention regroupent les pénicillines A (amoxicilline), les 5-nitro-imidazolés seuls ou associés aux macrolides, et, notamment en cas d'allergie aux B-lactamines, les macrolides, les streptogramines (pristinamycine) et les lincosamides.

L'association amoxicilline - acide clavulanique est recommandée en deuxième intention.

Les cyclines doivent être réservées au seul traitement de la parodontite juvénile localisée, même si d'autres antibiotiques peuvent être utilisés.

L'utilisation des céphalosporines n'est pas recommandée.

\subsubsection{Infections sévères}

Dans les infections sévères, en milieu spécialisé, on prescrira les mêmes familles d'antibiotiques par voie parentérale avec des adaptations de posologie selon le foyer et l'état fonctionnel. Les glycopeptides seront prescrits en cas d'allergie aux B-lactamines et/ou de résistance. L'utilisation des céphalosporines est possible en deuxième intention, après documentation microbiologique et antibiogramme.

\section{Antibiothérapie prophylactique}

\subsection{Indications de l'antibioprophylaxie en fonction du risque de l'acte bucco- dentaire}

\subsubsection{Actes invasifs}

Le risque infectieux est présent au cours de tous les actes invasifs (avec risque de saignement significatif) pratiqués chez les sujets à risque A (excepté la mise en place d'une digue pour laquelle il n'y a pas de risque infectieux chez les sujets à risque $A$ ) ou $B$, et au cours de certains de ces actes pratiqués chez les sujets sains. Une antibioprophylaxie est recommandée en cas d'acte à risque, que ce soit chez un sujet à risque ou chez un sujet sain (Accord professionnel).

Les actes invasifs pratiqués chez les sujets sains pour lesquels une antibioprophylaxie est recommandée sont définis comme étant : l'avulsion de dent en désinclusion, les transplantations/réimplantations, la chirurgie périapicale, la chirurgie des tumeurs bénignes des maxillaires, certains actes de chirurgie parodontale (comblement et greffes osseuses, membranes en chirurgie de la poche), la chirurgie osseuse, la mise en place en chirurgie implantaire et la mise en place de matériaux de comblement (Accord professionnel).

L'intérêt d'une antibioprophylaxie chez le sujet sain n'est pas reconnu au cours du traitement des dents à pulpe non vitale (y compris la reprise de traitement canalaire), des avulsions de dent infectée ou incluse et d'une germectomie (Accord professionnel).

Les actes invasifs pratiqués chez les sujets sains ne justifiant pas d'antibioprophylaxie sont définis comme étant : les anesthésies locales intraligamentaires, la mise en place d'une digue, le traitement des dents à pulpe vitale, les soins prothétiques à risque de saignement, les soins parodontaux non chirurgicaux, certaines avulsions dentaires (dent saine, alvéolectomie, séparation des racines, amputation radiculaire), la chirurgie des tumeurs bénignes des tissus mous, certains actes de chirurgie parodontale (lambeau d'accès en chirurgie de la poche, chirurgie mucogingivale), les freinectomies, le dégagement de stade II en chirurgie implantaire, et les actes d'orthopédie dento-faciale (Accord professionnel).

Les actes invasifs pratiqués chez les sujets à risque $A$ pour lesquels une antibioprophylaxie est recommandée sont définis comme étant : les soins endodontiques, les soins prothétiques à risque de saignement, et tous les actes chirurgicaux. \begin{tabular}{l}
$\begin{array}{l}\text { médecine } \\
\text { buccale } \\
\text { chirurgie } \\
\text { buccale }\end{array}$ \\
\hline VOL. $9, N^{\circ} 1$ \\
2003 \\
\hline page 53
\end{tabular} 
médecine

buccale

chirurgie

buccale

VOL. $9, \mathrm{~N}^{\circ} 1$ 2003

page 54
L'intérêt d'une antibioprophylaxie chez le sujet à risque A n'est pas reconnu au cours des anesthésies locales intraligamentaires et des soins parodontaux non chirurgicaux (Accord professionnel).

Les actes invasifs pratiqués chez les sujets à risque $B$ pour lesquels une antibioprophylaxie est recommandée sont définis comme étant : les anesthésies locales intraligamentaires, les soins prothétiques à risque de saignement, les soins parodontaux non chirurgicaux, la mise en place d'une digue.

Les soins endodontiques et les actes chirurgicaux nécessitent aussi une antibioprophylaxie, mais certains sont contre-indiqués chez ce type de sujet en raison d'un risque trop élevé : le traitement des dents à pulpe non vitale (y compris la reprise de traitement canalaire), l'amputation radiculaire, les transplantations/réimplantations, la chirurgie péri-apicale, la chirurgie parodontale, la chirurgie implantaire et la mise en place de matériaux de comblement (Accord professionnel).

\subsubsection{Actes non invasifs}

II n'y a pas de risque infectieux au cours d'actes non invasifs (sans risque de saignement significatif) pratiqués chez les sujets sains, ni chez les sujets à risque $A$ ou $B$; une antibioprophylaxie n'est donc pas justifiée dans ces cas-là (Accord professionnel).

Sont considérés comme actes non invasifs : les actes de prévention, les soins conservateurs, les soins prothétiques non sanglants, l'ablation post-opératoire de sutures, la pose de prothèses amovibles orthodontiques et la pose ou l'ajustement d'appareils orthodontiques, la prise de radiographies dentaires, et les anesthésies locales non intraligamentaires (Accord professionnel).

\subsection{Antibioprophylaxie des infections iatrogènes}

La prophylaxie standard de l'endocardite infectieuse, requiert une seule prise d'antibiotique par voie orale une heure avant le geste. On s'oriente actuellement vers une prescription d'une dose de $\mathbf{2} \mathbf{g}$ d'amoxicilline chez l'adulte, et de 50 mg. $\mathrm{kg}^{-1}$ chez l'enfant.

En cas d'allergie aux B-lactamines, il est recommandé de prescrire une dose de $600 \mathrm{mg}$ de clindamycine chez l'adulte, et de $15 \mathrm{mg} \cdot \mathrm{kg}^{-1}$ chez l'enfant. On peut également utiliser la pristinamycine à la dose de $1 \mathrm{~g}$ chez l'adulte, et de 25 mg. $\mathrm{kg}^{-1}$ chez l'enfant.

Quand la prophylaxie doit être administrée par voie parentérale, il est recommandé d'utiliser, dans l'heure précédant le geste, l'amoxicilline $2 \mathrm{~g}$ IV chez l'adulte et 50 mg. $\mathrm{kg}^{-1}$ IV chez l'enfant (perfusion de $30 \mathrm{~min}$ ), puis $1 \mathrm{~g}$ per os chez l'adulte et $25 \mathrm{mg} \cdot \mathrm{kg}^{-1}$ chez l'enfant, 6 heures plus tard.

En cas d'allergie aux ß-lactamines lorsque la prophylaxie doit être administrée par voie parentérale, un glycopeptide peut être utilisé, dans I'heure précédant le geste (vancomycine $1 \mathrm{~g}$ IV chez l'adulte et $20 \mathrm{mg} \cdot \mathrm{kg}^{-1} \mathrm{chez}$ l'enfant en perfusion d'au moins $60 \mathrm{~min}$, ou teicoplanine $400 \mathrm{mg}$ en injection IV directe uniquement chez l'adulte) (Accord professionnel).

La prophylaxie des infections sur prothèses articulaires est identique à celle de l'endocardite infectieuse (Accord professionnel).

Le traitement préventif des infections locales sera choisi en se référant au spectre antimicrobien des antibiotiques et des germes habituellement rencontrés, et à l'indication de l'association métronidazole - spiramycine (à une posologie de 4,5 M UI de spiramycine et de $750 \mathrm{mg}$ de métronidazole) (Accord professionnel).

Le traitement préventif des infections générales sera choisi en se référant au spectre antimicrobien des antibiotiques et des germes habituellement rencontrés, et sera administré par voie intraveineuse. L'association amoxicilline - acide clavulanique est recommandée à la posologie de $2 \mathrm{~g}$ d'amoxicilline en préopératoire ; l'association amoxicilline - métronidazole peut être également utilisée. En cas d'allergie aux B-lactamines, l'association clindamycine - gentamicine est recommandée (Accord professionnel). 


\section{Traitements adjuvants}

L'utilisation de l'antibiothérapie locale à libération immédiate n'est pas recommandée pour le traitement des infections bucco-dentaires (Accord professionnel).

L'utilisation de l'antibiothérapie locale à libération contrôlée n'a pas d'intérêt clairement démontré pour le traitement des parodontites
(Accord professionnel).

L'irrigation sous-gingivale d'antibiotique dans le cadre du traitement de la parodontite n'est pas recommandée (Accord professionnel).

Les anti-inflammatoires non-stéroïdiens, y compris l'aspirine, ne doivent pas être prescrits en première intention au cours des infections bucco-dentaires (Accord professionnel). 